OPEN ACCESS

Edited by:

Luiza Ghila

University of Bergen, Norway

Reviewed by:

Pei-Chun Chen,

National Cheng Kung

University, Taiwan

Tianhua Niu,

Tulane University, United States

*Correspondence:

Tuanyu Fang

fangtuanyu_2010@163.com

Specialty section:

This article was submitted to

Clinical Diabetes,

a section of the journal

Frontiers in Endocrinology

Received: 01 February 2020

Accepted: 07 August 2020

Published: 11 September 2020

Citation:

Lin L, Quan H, Chen K, Chen D, Lin D and Fang $T$ (2020) ABCC8-Related

Maturity-Onset Diabetes of the Young (MODY12): A Report of a Chinese Family. Front. Endocrinol. 11:645. doi: 10.3389/fendo.2020.00645

\section{ABCC8-Related Maturity-Onset Diabetes of the Young (MODY12): A Report of a Chinese Family}

\author{
Leweihua Lin, Huibiao Quan, Kaining Chen, Daoxiong Chen, Danhong Lin and \\ Tuanyu Fang*
}

Department of Endocrinology, Hainan General Hospital, Hainan Affiliated Hospital of Hainan Medical University, Haikou, China

Maturity-onset diabetes mellitus of the young (MODY) is a monogenic diabetes characterized by autosomal dominant inheritance. Its atypical clinical features make diagnosis difficult and it can be misdiagnosed as type 1 or type 2 diabetes. Fourteen subtypes of MODY have been diagnosed so far, of which MODY12 is caused by mutation of the ABCC8 (ATP Binding Cassette Subfamily C Member 8) gene, which is rarely reported in China. This paper reports a Chinese family of MODY12 caused by a rare missense mutation on the $A B C C 8$ gene, which has not been reported to be associated with MODY in China or in other countries, with the aim of increasing clinicians' awareness and attention to the disease.

Keywords: $A B C C 8$ gene, missense mutation, MODY, diabetes, metformin

\section{INTRODUCTION}

Monogenic diabetes refers to a specific type of diabetes resulting from monogenic mutation, of which the most common type is maturity-onset diabetes of the young (MODY). MODY is an autosomal dominant hereditary disease leading to dysfunction of the pancreatic $\beta$-cells. Since its discovery, mutations have been identified for at least these 14 genes (HNF4A, GCK, HNF1A, PDX1, HNF1B, NEUROD1, KLF11, CEL, PAX4, INS, BLK, ABCC8 [ATP Binding Cassette Subfamily C Member 8], KCNJ11, and APPL1) for MODY (1-3). A very high prevalence of family members carry the mutated gene, and patients in the same family have similar clinical manifestations. About $80 \%$ of MODY is previously misdiagnosed as type 1 or type 2 diabetes (4-6) because it is a relatively rare condition and there is low awareness of the clinical phenotype and availability of testing. Its atypical clinical features are the main cause of its misdiagnosis, and the diagnosis relies more on genetic testing, which is expensive for the average wage-earner and is not covered by all medical insurance institutions at present. Correct diagnosis of MODY is essential for optimizing treatment, prognosis, and genetic counseling. At present, related research on MODY is relatively scarce in China. Here, we report a family of MODY12, which is caused by a rare missense mutation.

\section{FAMILY}

The 1999 World Health Organization diagnostic criteria were used to diagnose diabetes mellitus (DM) or impaired glucose tolerance (IGT) (7). The family has a family history of three generations of diabetes (Figure 1). A total of nine people have been diagnosed with diabetes or impaired glucose 


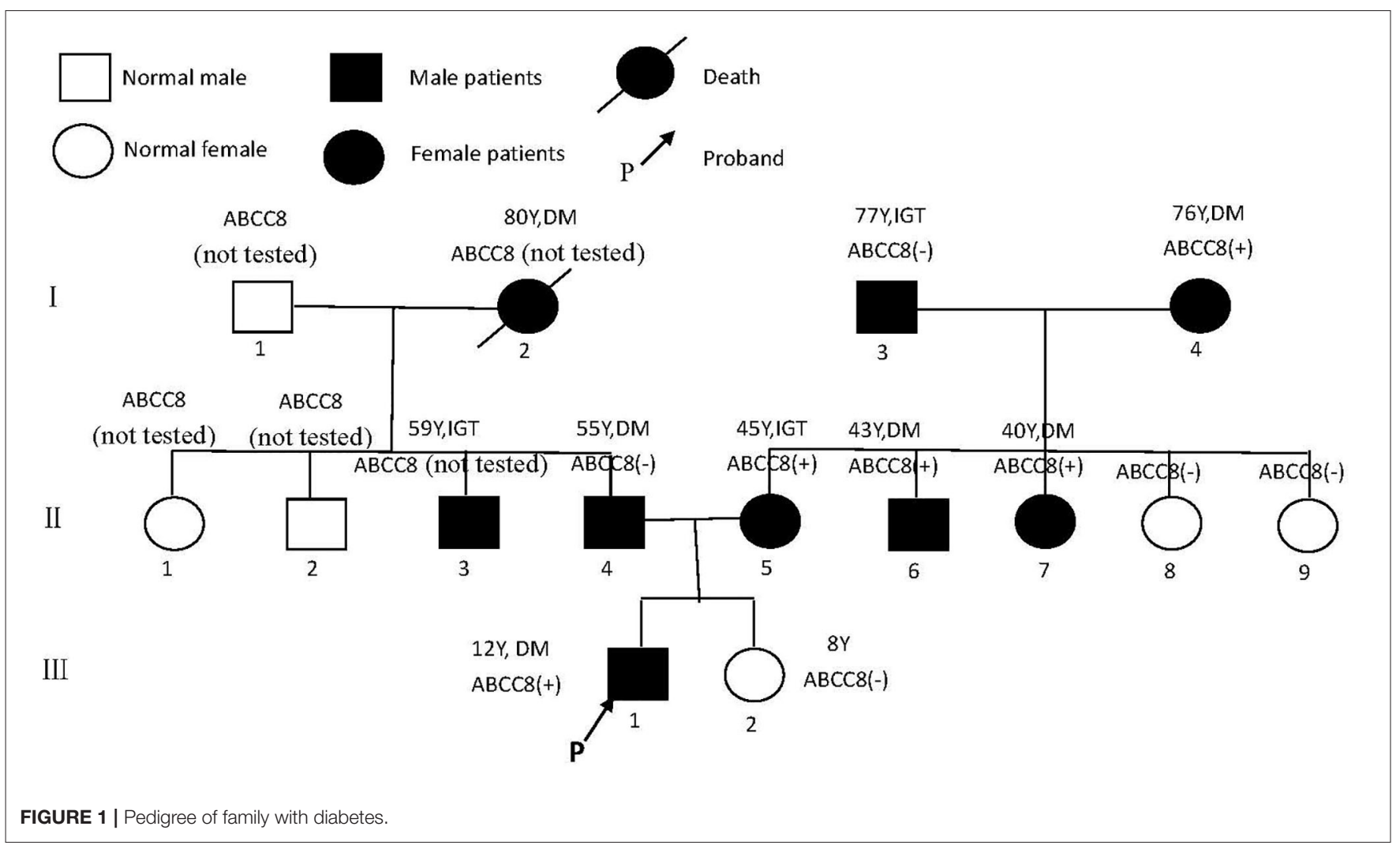

tolerance, of whom three had been diagnosed before the age of 45 years, and the earliest diagnosed age is 12 years old.

\section{The Proband (III1)}

Male, 12 years old, Han ethnicity. He was admitted to hospital on January 4, 2019, because of polyuria, polydipsia, polyphagia, weight loss for half a year, binocular photophobia, and lacrimation for 2 weeks. There were no obvious causes for the above symptoms, and he had accompanying weight loss of $11 \mathrm{~kg}$ for half a year, but had ignored it. Two weeks ago, because of binocular photophobia, tearing, pain, and blurred vision, he visited a local hospital. There, it was found that his fasting blood glucose was $17.1 \mathrm{mmol} / \mathrm{L}$ and that he was positive for urine ketone bodies, which was considered type 1 diabetes viral keratitis. He was transferred to our hospital for further treatment.

\section{Personal History}

He was the first child of a 25-year-old woman with spontaneous term delivery at week 34 of an uneventful pregnancy; his birth weight was $2,700 \mathrm{~g}$. When he was 3 months old, he received hyperbaric oxygen therapy for 4 months because of motor system developmental delay. At 1 year old, his growth and development were satisfactory as children of the same age. His intelligence is normal.

\section{Family History}

His grandmother was hospitalized when she was 80 years old for pancreatic cancer, where it was found that her blood sugar was elevated, but she eventually died from respiratory failure caused by infection.

\section{Physical Examination}

Height, $173 \mathrm{~cm}$; weight, $70 \mathrm{~kg}$; body mass index (BMI), 23.39 $\mathrm{kg} / \mathrm{m}^{2}$, regular pulse (86 beats per $\mathrm{min}$ ), blood pressure (BP), 125/79 mmHg; waist circumference, $83 \mathrm{~cm}$. He had binocular conjunctival hyperemia and edema. He had no buffalo hump, moon face, skin purple striae, or centripetal obesity. Cardiopulmonary examination showed no abnormality. Biochemical analysis revealed normal liver and kidney function, triglyceride (TG) of $1.16 \mathrm{mmol} / \mathrm{L}$, total cholesterol (CHOL) of $5.82 \mathrm{mmol} / \mathrm{L}$, and low-density lipoprotein cholesterol (LDL-C) of $4.04 \mathrm{mmol} / \mathrm{L}$. His glycated hemoglobin A1c (HbA1c) level was $13.0 \%$. C-peptide $(0 \mathrm{~min})$ was $0.438 \mathrm{nmol} / \mathrm{L}$ (chemiluminescent; reference range, $0.37-1.47 \mathrm{nmol} / \mathrm{L}$ ), and C-peptide (120 min) was $1.440 \mathrm{nmol} / \mathrm{L}$. He was negative for anti-glutamic acid decarboxylase (GAD) and anti-insulin autoantibodies. Urine microalbumin was $8.0 \mathrm{mg} / \mathrm{L}$ (reference range, 0-30 mg/L). Color ultrasound revealed left popliteal artery intimal-medial thickness and atherosclerotic plaque formation at the proximal part of the right subclavian artery. The optic fundi revealed no hemorrhages or exudates, and the fundal vessels were unremarkable. Neuroelectromyography was normal. He was put on combination therapy with basal (glargine) insulin and recombinant human insulin (RI) to control blood sugar; antiviral eyedrops and other treatments improved his condition. After 1 week, his glycemic control had improved remarkably, 
with the vast majority of both fasting (4.2-6.1 mmol/L) and postprandial $(6.2-10.0 \mathrm{mmol} / \mathrm{L})$ glucose values within the target range. He had no hypoglycemia reaction, and his review for urine ketone bodies was negative. For better compliance, we changed the insulin combination therapy to mixed recombinant human insulin lispro injection (50R) (12 U daily) after discharge. After 4 months, his body weight gradually increased by about $8 \mathrm{~kg}$ (height, $176 \mathrm{~cm}$ ). On May 16, 2019, we reviewed blood sugar (BS, 0-60-120 min) (no hypoglycemic drugs): 6.10-9.60$11.0 \mathrm{mmol} / \mathrm{L}$, and C-peptide (0-60-120 min): 1.530-3.080-3.130 $\mathrm{nmol} / \mathrm{L}$. Biochemical analysis revealed growth hormone of $3.490 \mathrm{ng} / \mathrm{ml}$ (reference range, $0.077-10.8 \mathrm{ng} / \mathrm{ml}$ ). Therefore, we attempted to discontinue insulin therapy and started treatment with $1.0 \mathrm{~g} /$ days metformin combined with diet and exercise therapy because of overweight. His glucose fluctuations were within the $6-10 \mathrm{mmol} / \mathrm{L}$ range. Reviewing his medical history and adolescent onset, his ketosis had a predisposing factor of viral keratitis, his blood sugar was easily controlled, he had good islet $\beta$-cell function, and he was negative for anti-GAD and antiinsulin autoantibodies. Based on the above, MODY was highly suspected. Accordingly, we conducted further investigations on his family.

\section{The Proband's Father (II4)}

$\mathrm{He}$ is 55 years old; Han ethnicity. BMI, $28.03 \mathrm{~kg} / \mathrm{m}^{2}$; waist circumference, $96 \mathrm{~cm}$. BP, 135/82 $\mathrm{mmHg}$. Oral glucose tolerance test (OGTT, 0-120 min): BS, 6.1-11.8 mmol/L; C-peptide, 1.010$4.200 \mathrm{nmol} / \mathrm{L}$. HbA1c, 6.3\%. TG, $2.20 \mathrm{mmol} / \mathrm{L}$; CHOL, 5.51 $\mathrm{mmol} / \mathrm{L}$; LDL-C, $1.30 \mathrm{mmol} / \mathrm{L}$; liver and kidney function, normal. At present, he is on diet and exercise treatments; his blood sugar is well-controlled.

\section{The Proband's Mother (II5)}

She is 45 years old; Han ethnicity. BMI, $23.18 \mathrm{~kg} / \mathrm{m}^{2}$; waist circumference, $78 \mathrm{~cm}$. BP, 115/75 mmHg. OGTT (0-120 min): BS, 4.3-10.6 mmol/L; C-peptide, 1.000-4.150 nmol/L. HbA1c, $5.4 \%$. At present, her diabetes is treated with diet and exercise; her blood sugar is well-controlled.

\section{The Proband's Grandmother (I2)}

Deceased; Han ethnicity. When she was 80 years old, she was found to have high blood sugar due to duodenal tubulopapillary adenocarcinoma (late stage). She was not examined further, and was not treated with anti-diabetic therapy. She subsequently died from obstructive jaundice, metabolic encephalopathy, and multiple organ failure.

\section{The Proband's Uncle (II3)}

$\mathrm{He}$ is 59 years old; Han ethnicity. BMI, $29.75 \mathrm{~kg} / \mathrm{m}^{2}$; waist circumference, $98 \mathrm{~cm}$. BP, 135/82 mmHg. OGTT (0-120 min): BS, 5.2-8.6 mmol/L; C-peptide, 1.020-4.760 nmol/L. HbAlc, $6.2 \%$. At present, his diabetes is treated with diet and exercise; his blood sugar is well-controlled.

\section{The Proband's Uncle (II6)}

$\mathrm{He}$ is 48 years old; Han ethnicity. He was diagnosed with diabetes 5 years ago, and his diabetes is treated irregularly with glipizide; his blood sugar is generally under control, with no spontaneous ketosis tendency. BMI, $23.18 \mathrm{~kg} / \mathrm{m}^{2}$; waist circumference, $82 \mathrm{~cm}$. BP, 120/78 mmHg. He refused examination for $\mathrm{HbAlc}$ and $\mathrm{C}$-peptide.

\section{The Proband's Aunt (II7)}

She is 40 years old; Han ethnicity. BMI, $22.60 \mathrm{~kg} / \mathrm{m}^{2}$; waist circumference, $73 \mathrm{~cm}$. BP, $110 / 70 \mathrm{mmHg}$. OGTT (0-120 min): BS, 3.6-11.2 mmol/L; C-peptide, 0.571-3.23 nmol/L. HbA1c, $5.5 \%$. At present, her diabetes is treated with diet and exercise; her blood sugar is well-controlled.

\section{The Proband's Grandmother (I4)}

She is 76 years old; Han ethnicity. She has a history of sequelae of cerebral infarction and hypertension for 5 years. BMI, 18.26 $\mathrm{kg} / \mathrm{m}^{2}$; waist circumference, $69 \mathrm{~cm}$. BP, $110 / 80 \mathrm{mmHg}$. OGTT (0-120 min): BS. 4.7-13.2 mmol/L; C-peptide, 0.59-4.51 nmol/L. HbA1c, 5.4\%. At present, her diabetes is treated with diet and exercise to control blood sugar and with amlodipine to control $\mathrm{BP}$, both of which are well-controlled.

\section{The Proband's Grandfather (I3)}

He is 77 years old; Han ethnicity. He has had hypertension for 10 years. BMI, $25.96 \mathrm{~kg} / \mathrm{m}^{2}$; waist circumference, $92 \mathrm{~cm}$. BP, 140/80 mmHg. OGTT (0-120 min): BS, 4.4-9.0 mmol/L; C-peptide, 0.488-3.67 nmol/L. HbA1c, 5.4\%. At present, his diabetes is treated with diet and exercise; his blood sugar is well-controlled.

\section{Genetic Analysis}

The combination of negative autoantibodies for type 1 diabetes with inappropriately low C-peptide levels and a family history of diabetes or abnormal glucose tolerance prompted the molecular investigation for MODY. After obtaining informed consent, a venous blood ( $5 \mathrm{ml}$ ) was collected from the proband (III1) as well as his families and placed in an anti-coagulant tube containing ethylenediaminetetraacetic acid (EDTA).

DNA was extracted from the venous blood collected from the patient as well as from the patient's families using the QIAamp DNA Blood Midi kit (Qiagen, Germany), The quantity/quality of the DNA preparations was assessed using a NanoDrop 1000 spectrophotometer (Thermo Fisher, USA).

Exome enrichment was performed using the IDT xGenExome ResearchPanelv1.0 (Integrated DNA Technologies, Coralville, lowa, USA) and 150 base pair, paired end sequencing was performed using an Illumina HiSeq 4000 platform (San Diego, CA, USA). Mean sequencing depth and nucleotides with $>20 \mathrm{X}$ sequencing depth were $159 \mathrm{x}$ and $99.61 \%$, respectively. The raw sequencing reads were aligned by the We-Health BioMedical Technology (Shanghai, China) using the BurrowsWheeler Aligner (BWA) and SAMtools. Polymorphic variants that showed $>0.5 \%$ allele frequency in general populations were filtered out (based on the 1000 genomes database). The SIFT, PolyPhen2, MutationTaster were used to assist in predicting the functional impact of identified missense variants $(8,9)$.

The next-generation sequencing data were analyzed for mutations in known MODY-related gene (included the genes for HNF4A, GCK, HNF1A,PDX1, HNF1B, NEUROD1, KLF11, CEL, PAX4, INS, BLK, ABCC8, KCNJ11, and APPL1). The 
sequencing data revealed a heterozygous missense mutation: c.C4544T (p.T1515M), in exon 37 of the ABCC8 gene of the proband (III1) that was derived from his mother (II5). The amino acid changed from threonine to methionine (Figure 2); no mutations were detected for the remaining 13 genes. For the heterozygous mutation c.C4544T (p.T1515M) of the ABCC8 gene detected in III1, we verified I3, I4, II4, II5, II6, II7, II8, II9, III1, and III2 using Sanger sequencing and found that III1, II5, II6, II7, and I4 all had the same mutation of the $A B C C 8$ gene.

Oligonucleotides flanking the genomic locations of identified variantswere designed using the Primer3Plus browser. Polymerase chainreactions (PCR) was performed with the following primers: 5'-CACCCCACAGGACTGAACAG$3^{\prime}$ and $5^{\prime}-$ ATCTGCTCCACTCACAGCAC- $3^{\prime}$. PCR products were sequenced bi-directionally using Big Dye Terminatorchemistry v3.1 and sequenced using an $\mathrm{ABI}$ 3730XL sequencer (AppliedBiosystems/Life Technologies, Carlsbad, CA, USA). Sequences were reviewed manually and compared using CodonCode Aligner to reference sequences: $A B C C 8$ (NM_000352).

This study was approved by the Ethics Committee of Hainan General Hospital. All subjects gave written informed consent in accordance with the Declaration of Helsinki.

\section{DISCUSSION}

Monogenic diabetes is a special type of diabetes caused by a single gene mutation or defect; it is genetically and clinically heterogeneous, and includes MODY, neonatal diabetes, congenital hyperinsulinemia, and Wolcott-Rallison syndrome (10). First-degree relatives of a MODY family member carrying genetic mutations for the 2 most common MODY subtypes, i.e., GCK-MODY2 or HNF1 $\alpha-$ MODY3, could have $95 \%$ risk of developing diabetes (11), and patients in the same family are more likely to have similar clinical presentations. MODY is the most common form of monogenic diabetes $(1-3,12,13)$. Previous studies agree that the diagnostic criteria of MODY are: (i) autosomal dominant inheritance, (ii) insulin independence within 2 years of onset, (iii) at least one family member diagnosed with diabetes before the age of 25 years, (iv) combined with islet $\beta$-cell dysfunction (14).

The identification of a MODY subtype is crucial for the choice of adequate treatment. However, with the maturing of genetic testing technology in recent years, an increasing number of patients with MODY discovered by genetic testing do not fully meet the above diagnostic criteria, and their clinical manifestations tend to be more diverse. Strict adherence to the above criteria may result in missed diagnosis of a large number of patients with MODY. A study from the UK has shown that a significant number of patients with MODY have a primary diagnosis of diabetes and the MODY diagnosis could be confirmed only by performing molecular genetic testing (15), which may lead to inappropriate treatment such as insulin and/or insulin sensitization treatment (16). Therefore, when the onset of diabetes in young patients is not typical type 1 or type 2 diabetes, it should prompt molecular investigation for MODY.
The ABCC8 gene is located on chromosome 11p15.1 and encodes the sulfonylurea receptor 1 (SUR1) subunit of the ATP-sensitive potassium $\left(\mathrm{K}_{\mathrm{ATP}}\right)$ channel in the pancreatic $\beta$ cell, which is involved in the electrical activity of the plasma membrane, thereby regulating insulin secretion (17). ABCC8 gene mutations can cause a variety of phenotypes, resulting in overactivity or underactivity of the $\mathrm{K}_{\mathrm{ATP}}$ channel, resulting in abnormal glucose metabolism. In 2012, Bowman et al. first reported that MODY12 is caused by $A B C C 8$ gene mutation, and its clinical manifestations are diverse, may be associated with overweight or obesity, and are usually with no significant hypertriglyceridemia and hypercholesterolemia. Further, such families may also have neonatal diabetic patients $(18,19)$. As sulfonylureas specifically bind to the SUR1 subunit and shut down the channel to release insulin in a non-ATP-dependent manner, this type of MODY is sensitive to sulfonylureas.

Initially, the present case was misclassified with type 1 diabetes because of his young age and ketosis, despite the negative islet $\beta$-cell autoantibodies. The possibility of considering the case as MODY was supported by the presence of diabetes within three generations, the stable C-peptide levels, and the obvious signs of overdose on relatively small insulin doses, which led to overweight. The characteristics of the family are: the proband's age is $<25$ years old with insulin deficiency, and his ketosis had precipitating factors and was easily eliminated. The diabetes-related antibodies (islet cell antibody [ICA], tyrosine phosphatase-like protein antigen-2 antibody [IA2A], glutamic acid decarboxylase antibody [GADA]) were negative, and a follow-up visit after 4 months on the regimen showed that his glycemic control had remarkably improved, with the vast majority of both fasting and postprandial glucose values within the target range and $\mathrm{HbAlc}$ of $5.7 \%$; islet $\beta$-cell function was significantly improved, and even the overweight and insulin resistance appeared. All three generations in the family have diabetes or impaired glucose tolerance with autosomal dominant inheritance. In summary, our evidence supports the diagnosis of MODY. Genetic testing verified the presence of the $A B C C 8$ gene mutation (ABCC8: NM_000352: exon37: c.C4544T: p.T1515M) in the proband (III1), his mother (II5) and three maternal relatives (II6, II7, I4) with diabetes or impaired glucose tolerance with similar figures and islet $\beta$-cell function, but not in the other relatives (II8, II9, III1) who do not have diabetes or impaired glucose tolerance, suggesting that the above mutation and diabetes have obvious co-segregation in the family. In addition, the proband's uncle (II6) had early-onset diabetes and took glipizide for it without spontaneous ketosis. This mutation (ABCC8: NM_000352: exon37: c.C4544T: p.T1515M) can lead to the conserved amino acid residues of this site being replaced by different amino acids throughout the evolution process, resulting in congenital hyperinsulinemia (20), and it has been suggested that congenital hyperinsulinemia caused by $A B C C 8$ gene mutation can develop into MODY $(21,22)$, but since then, there has been no report of MODY12 caused by the above mutation. The SIFT score, PolyPhen 2 score, and Mutation Taster score was 0,1 , and 1 , all suggesting that it is damaging to the protein function. Based on the above predictions, the mutation is considered pathogenic. Therefore, we speculate that 


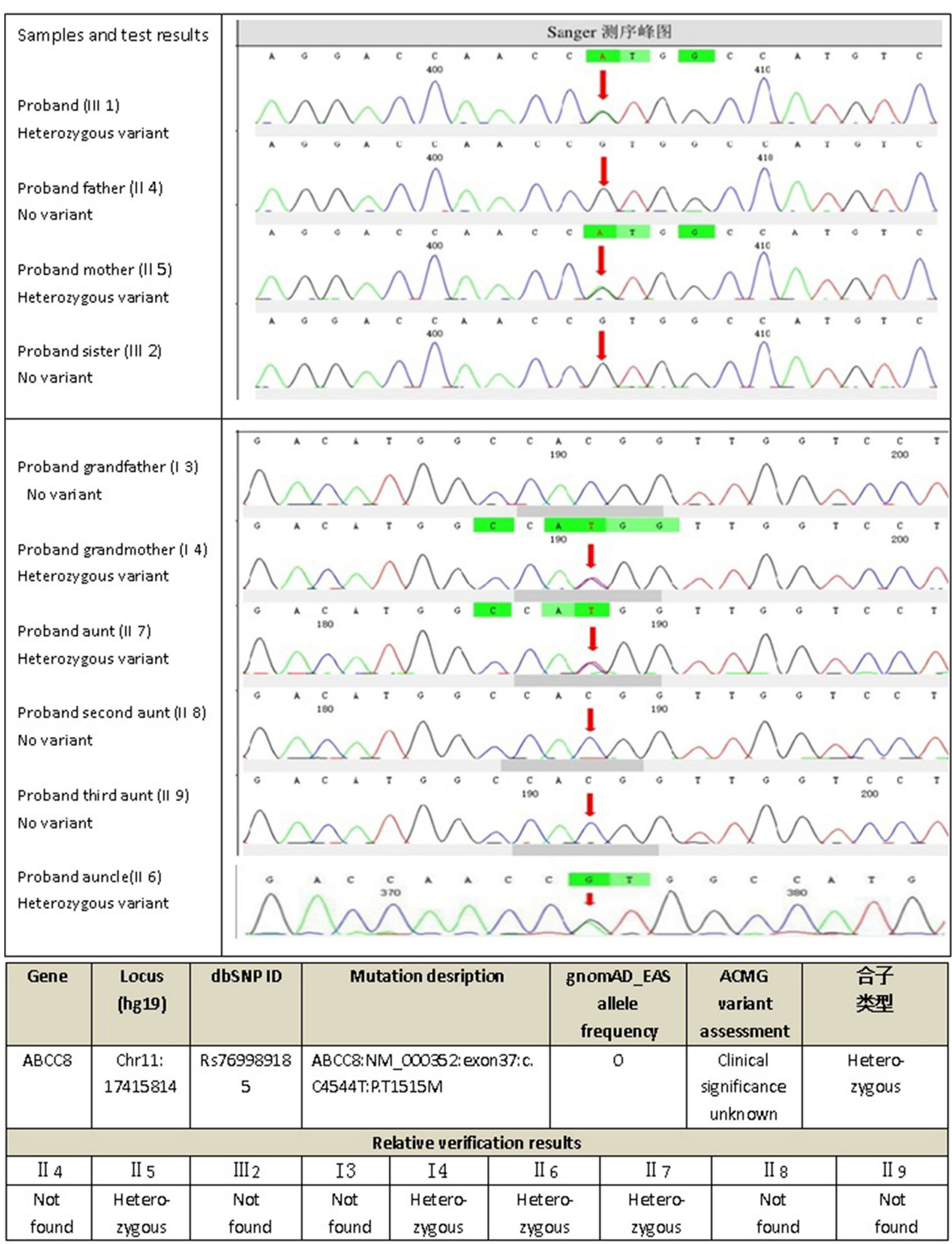

FIGURE 2 | Gene_sequencing results of proband and his family. 
TABLE 1 | Clinical characteristics of family members with disease.

\begin{tabular}{|c|c|c|c|c|c|c|c|c|}
\hline & Gender & $\begin{array}{l}\text { Age at diagnosis } \\
\text { (years) }\end{array}$ & $\begin{array}{c}\text { BMI } \\
\left(\mathrm{kg} / \mathrm{m}^{2}\right)\end{array}$ & $\begin{array}{l}\text { Waistline } \\
\text { (cm) }\end{array}$ & $\begin{array}{c}\text { 0'BS } \\
\text { (mmol/L) }\end{array}$ & $\begin{array}{l}\text { 120'BS } \\
\text { (mmol/L) }\end{array}$ & $\begin{array}{c}0^{\prime} \mathrm{CP} \\
\text { (nmol/L) }\end{array}$ & $\begin{array}{c}\text { HbA1c } \\
(\%)\end{array}$ \\
\hline III1 & $\mathrm{M}$ & 12 & 23.39 & 83 & 9.0 & 18.8 & 0.438 & 13.0 \\
\hline 115 & $\mathrm{~F}$ & 45 & 23.18 & 78 & 4.3 & 10.6 & 0.488 & 5.4 \\
\hline 116 & $\mathrm{M}$ & 43 & 23.18 & 82 & - & - & - & - \\
\hline 117 & $\mathrm{~F}$ & 40 & 22.60 & 73 & 3.6 & 11.2 & 0.571 & 5.5 \\
\hline 14 & $F$ & 76 & 18.26 & 69 & 4.7 & 13.2 & 0.59 & 5.4 \\
\hline 114 & $\mathrm{M}$ & 55 & 28.03 & 96 & 6.1 & 11.8 & 1.020 & 6.3 \\
\hline 113 & $\mathrm{M}$ & 59 & 29.75 & 98 & 5.2 & 8.6 & 1.010 & 6.2 \\
\hline 12 & $\mathrm{~F}$ & 80 & - & - & - & - & - & - \\
\hline 13 & $\mathrm{M}$ & 77 & 25.96 & 92 & 4.4 & 9.0 & 1.000 & 5.4 \\
\hline
\end{tabular}

BMI, body mass index; BS, blood sugar; CP, C-peptide; HbA1C, glycated hemoglobin A1C. The "-" indicates not available.

this mutation site is the cause of diabetes or impaired glucose tolerance in the proband (III1), his mother (II5), and the three maternal relatives (II6, II7, I4), where the type of diabetes is considered MODY12. I3, II3, and II4 also have diabetes or impaired glucose tolerance, but unlike the proband, they do not have the above mutation, and have characteristics such as central obesity or overweight, insulin resistance, diabetes diagnosed at age $>50$ years, and whose diagnosis is more prone to type 2 diabetes, which are quite different from the proband (Table 1).

The prevalence of MODY subtypes varies widely between countries and ethnic groups. More than $80 \%$ of Caucasian patients with MODY are MODY3 or MODY2 $(15,23)$, but only $7.2-36.7 \%$ of Asian patients with MODY are diagnosed as MODY2 or MODY3 (24-27). In China, epidemiological research of MODY is in its infancy, and the distributions of different MODY subtypes are not so clear. Further genes related to MODY are likely to be found, as most patients with MODY have unknown mutations; this group is defined as MODYX. MODYX might be responsible for $80-90 \%$ of MODY in China, which is quite different from other populations $(25,26,28,29)$. A lack of awareness of MODY, as well as its similar clinical features to other types of diabetes, means that clinicians cannot often easily distinguish MODY from type 1 or type 2 diabetes without genetic testing, which may lead to misdiagnosis. In the present case, the proband was diagnosed with type 1 diabetes due to early onset (12 years old) with ketosis, the obvious signs of overweight on relatively small doses of insulin, significantly improved islet $\beta$ cell function, and even the appearance of insulin resistance. After family investigation and genetic testing, we revised the diagnosis of type 1 diabetes to MODY12, and attempted to discontinue insulin therapy and started treatment with $1.0 \mathrm{~g} /$ day metformin combined with diet and exercise therapy. The proband's blood glucose is well-controlled, and he has not gained any more weight. His relatives (II5, II6, II7, I4) with the same $A B C C 8$ gene mutation as the proband have similar habius, no central obesity, BMI within the $18-24 \mathrm{~kg} / \mathrm{m}^{2}$ range, mildly elevated blood glucose, and similar islet $\beta$-cell function. At present, they only require diet and exercise therapy. Therefore, the identification of a MODY subtype is crucial for the choice of adequate treatment. In the present case, verification of the diagnosis by genetic testing enabled the discontinuation of insulin therapy, which had produced obvious adverse effects, such as increased bodyweight.
In conclusion, we report a case of MODY12 caused by a single nucleotide mutation of cytosine to thymine at position 4,544 of the $A B C C 8$ gene. This mutation has not been reported to be associated with MODY in China or in other countries. It is a rare missense ABCC8 gene mutation (dbSNP ID: rs769989185, and has not been reported in ClinVar database: https://www.ncbi. nlm.nih.gov/clinvar/), which might be useful for individualized selection of appropriate treatment and for genetic consulting. The described case also highlights the clinical expression of diabetes related to the $A B C C 8$ gene and underlines the value of genetic testing in young patients presenting with nonautoimmune diabetes.

\section{DATA AVAILABILITY STATEMENT}

This article contains previously unpublished data. The name of the repository and accession number(s) are not available.

\section{ETHICS STATEMENT}

Written informed consent was obtained from the individuals and minor's legal guardian for the publication of any potentially identifiable images or data included in this article.

\section{AUTHOR CONTRIBUTIONS}

LL collected, analyzed, and interpreted the patient data and was the major contributor to the writing of the manuscript. TF made substantial contributions to the data interpretation, manuscript revision, and conducted the work. HQ helped with manuscript revision in English. HQ, KC, DC, and DL assisted with the patient data analysis. All authors are in agreement with the contents of the manuscript.

\section{FUNDING}

We are grateful for the support of the Hainan Province family planning science and education health project (no. 19A200034) and the major research and development program of Hainan Province (nos. ZDYF2018130 and ZDYF2019156). Lastly, the authors are deeply grateful to the proband and his family for their support. 


\section{REFERENCES}

1. Urakami T. Maturity-onset diabetes of the young (MODY): current perspectives on diagnosis and treatment. Diabetes Metab Syndr Obes. (2019) 12:1047-56. doi: 10.2147/DMSO.S179793

2. Oliveira SC, Neves JS, Pérez A, Carvalho D. Maturity-onset diabetes of the young: from a molecular basis perspective toward the clinical phenotype and proper management. Endocrinol Diabetes Nutr. (2020) 67:137-47. doi: 10.1016/j.endien.2020.03.001

3. Peixoto-Barbosa R, Reis AF, Giuffrida FMA. Update on clinical screening of maturity-onset diabetes of the young (MODY). Diabetol Metab Syndr. (2020) 12:50. doi: 10.1186/s13098-020-00557-9

4. Thomas ER, Brackenridge A, Kidd J, Kariyawasam D, Carroll P, Colclough $\mathrm{K}$, et al. Diagnosis of monogenic diabetes: 10-Year experience in a large multi-ethnic diabetes center. J Diabetes Investig. (2016) 7:332-7. doi: $10.1111 /$ jdi.12432

5. McDonald TJ, Ellard S. Maturity onset diabetes of the young:identification and diagnosis. Ann Clin Biochem. (2013) 50(Pt 5):403-15. doi: $10.1177 / 0004563213483458$

6. Thanabalasingham G, Pal A, Selwood MP. Systematic assessment of etiology in adults with a clinical diagnosis of young-onset type 2 diabetes is a successful strategy for identifying maturity-onset diabetes of the young. Diabetes Care. (2012) 35:1206-12. doi: $10.2337 / \mathrm{dc} 11-1243$

7. Department of Noncommunicable Disease Surveillance. Definition, Diagnosis, and Classification of Diabetes Mellitus and Its Complications: Report of a WHO Consultation. Part 1. Diagnosis and Classification of Diabetes Mellitus. Geneva: World Health Organization (1999).

8. Schwarz JM, Cooper DN, Schuelke M, Seelow D. MutationTaster2: mutation prediction for the deep-se quencing age. Nat Methods. (2014) 11:361-2. doi: $10.1038 /$ nmeth. 2890

9. Vaser R, Adusumalli S, Leng SN, Sikic M, Ng PC. SIFT missense predictions for genomes. Nat Protoc. (2016) 11:1-9. doi: 10.1038/nprot.2015.123

10. Kleinberger JW, Pollin TI. Undiagnosed MODY: time for action. Curr Diab Rep. (2015) 15:110. doi: 10.1007/s11892-015-0681-7

11. Heuvel-Borsboom H, de Valk HW, Losekoot M, Westerink J. Maturity onset diabetes of the young: Seek and you will find. Neth J Med. (2016) 74:193-200.

12. Glaser B. Insulin mutations in diabetes: the clinical spectrum. Diabetes. (2008) 57:799-800. doi: 10.2337/db08-0116

13. Li J, Sun S, Wang X, Li Y, Zhu H, Zhang H, et al. A missense mutation in IRS1 is associated with the development of early-onset type 2 diabetes. Int $J$ Endocrinol. (2020) 2020:9569126. doi: 10.1155/2020/9569126

14. Ellard S, Bellanne-Chantelot C, Hattersley AT. Best practice guidelines for the molecular genetic diagnosis of maturity-onset diabetes of the young. Diabetologia. (2008) 51:546-53. doi: 10.1007/s00125-008-0942-y

15. Shields BM, Hicks S, Shepherd MH, Colclough K, Hattersley AT, Ellard S. et al. Maturity-onset diabetes of the young(MODY):how many cases are we missing? Diabetologia. (2010) 53:2504-8. doi: 10.1007/s00125-010-1799-4

16. Chambers C, Fouts A, Dong F, Colclough K, Wang Z, Batish SD, et al. Characteristics of maturity onset diabetes of the young in a large diabetes center. Pediatr Diabetes. (2016) 17:360-7. doi: 10.1111/pedi.12289

17. Patch AM, Flanagan SE, Boustred C, Hattersley AT, Ellard S. Mutations in the ABCC8 gene encoding the SUR1 subunit of the KATP channel cause transient neonatal diabetes, permanent neonatal diabetes or permanent diabetes diagnosed outside the neonatal period. Diabetes Obes Metab. (2007) 9 (Suppl. 2):28-39. doi: 10.1111/j.1463-1326.2007. 00772.x
18. Bowman P, Flanagan SE, Edghill EL, Damhuis A, ShepherdMH, Paisey R, et al. Heterozygous ABCC8 mutations are a cause of MODY. Diabetologia. (2012) 55:123-7. doi: 10.1007/s00125-011-2319-x

19. Hartemann-Heurtier A, Simon A, Bellanne-Chantelot C, Reynaud R, Cavé $\mathrm{H}$, Polak M, et al. Mutations in the ABCC8 gene can cause autoantibodynegative insulin-dependent diabetes. Diabetes Metab. (2009) 35:233-35. doi: 10.1016/j.diabet.2009.01.003

20. Banerjee I, Skae M, Flanagan S E, Rigby L, Patel L, Didi M, et al. The contribution of rapid K ATP channel gene mutation analysis to the clinical management of children with congenital hyperinsulinism. Eur J Endocrinol. (2011) 164:733-40. doi: 10.1530/EJE-10-1136

21. Abdulhadi-Atwan M, Bushman J, Tornovsky-Babaey S, Perry A, Abu-Libdeh A, Glaser B, et al. Novel de novo mutation in sulfonylurea receptor 1 presenting as hyperinsulinism in infancy followed by oven diabetes in early adolescence. Diabetes. (2008) 57:1935-40. doi: 10.2337/db08-0159

22. Kapoor RR, Flanagan SE, James C, Shield J, Ellard S, Hussain K, et al. Hyperinsulinaemic hypoglycaemia. Arch Dis Child. (2009) 94:450-7. doi: $10.1136 /$ adc.2008.148171

23. Frayling TM, Evans JC, Bulman MP, Pearson E, Allen L, Owen K, et al. Beta-cell genes and diabetes: molecular and clinical characterization of mutations in transcription factors. Diabetes. (2001) 50 (Suppl. 1):S94-S100. doi: $10.2337 /$ diabetes.50.2007.S94

24. Yorifuji T, Fujimaru R, Hosokawa Y, Tamagawa N, Shiozaki M, Aizu K, et al. Comprehensive molecular analysis of Japanese patients with pediatriconset MODY -type diabetes mellitus. Pediatr Diabetes. (2012) 13:26-32.16. doi: 10.1111/j.1399-5448.2011.00827.x

25. Hwang JS, Shin $\mathrm{CH}$, Yang SW, Jung SY, Huh N. Genetic and clinical characteristics of Korean maturity-onset diabetes of the young (MODY) patients. Diabetes Res Clin Pract. (2006) 74:75-81. doi: 10.1016/j.diabres.2006.03.002

26. Mohan V, Radha V, Nguyen TT, Stawiski EW, Pahuja KB, Goldstein LD, et al. Comprehensive genomic analysis identifies pathogenic variants in maturityonset diabetes of the young (mody) patients in south india. BMC Med Genet. (2018) 19:22. doi: 10.1186/s12881-018-0528-6

27. Xu JY, Dan QH, Chan V, Wat NMS, Tam S, Tiu SC, et al. Genetic and clinical characteristics of maturity-onset diabetes of the young in Chinese patients. Eur J Hum Genet. (2005) 13:422-7. doi: 10.1038/sj.ejhg.5201347

28. Hu C, Jia W. Diabetes in china: epidemiology and genetic risk factors and their clinical utility in personalized medication. Diabetes. (2018) 67:3-11. doi: $10.2337 / \mathrm{dbi17}-0013$

29. Zhang M, Zhou JJ, Cui W, Li Y, Yang P, Chen X, et al. Molecular and phenotypic characteristics of matutity-onset diabetes of the young compared with early onset type 2 diabetes in China. J Diabetes. (2015) 7:858-63. doi: $10.1111 / 1753-0407.12253$

Conflict of Interest: The authors declare that the research was conducted in the absence of any commercial or financial relationships that could be construed as a potential conflict of interest.

Copyright $\odot 2020$ Lin, Quan, Chen, Chen, Lin and Fang. This is an open-access article distributed under the terms of the Creative Commons Attribution License (CC $B Y)$. The use, distribution or reproduction in other forums is permitted, provided the original author(s) and the copyright owner(s) are credited and that the original publication in this journal is cited, in accordance with accepted academic practice. No use, distribution or reproduction is permitted which does not comply with these terms. 